\title{
Relação entre Perfil de Densidade e Ligação Interna de Painéis OSB de Pinus spp
}

\author{
Paula Gabriella Surdi ${ }^{1}$, Geraldo Bortoletto Jr. ${ }^{2}$, Vinicius Resende Castro ${ }^{1}$, \\ Rafael Farinassi Mendes ${ }^{3}$, Natalie Ferreira de Almeida ${ }^{4}$, Mario Tomazello Filho ${ }^{5}$ \\ ${ }^{1}$ Departamento de Ciências Florestais, Escola Superior de Agricultura Luiz de Queiroz - ESALQ, \\ Universidade de São Paulo - USP, Piracicaba/SP, Brasil \\ ${ }^{2}$ Departamento de Ciências Florestais - LCF, Universidade de São Paulo - USP, Piracicaba/SP, Brasil \\ ${ }^{3}$ Departamento de Ciências Florestais, Universidade Federal de Lavras - UFLA, Lavras/MG, Brasil \\ ${ }^{4}$ Departamento de Ciências Florestais, Universidade de São Paulo - USP, Piracicaba/SP, Brasil \\ ${ }^{5}$ Departamento de Ciências Florestais, Escola Superior de Agricultura Luiz de Queiroz - ESALQ, \\ Universidade de São Paulo - USP, Piracicaba/SP, Brasil
}

\begin{abstract}
RESUMO
O presente trabalho teve por objetivos determinar a densidade aparente de painéis OSB de Pinus taeda e Pinus elliottii var. elliottii $\times$ Pinus caribaea var. hondurensis pelo método gravimétrico e pela densitometria de raios $\mathrm{X}$, obter os perfis densitométricos e verificar sua correlação com a propriedade mecânica de ligação interna. A densidade aparente dos painéis foi determinada pela densitometria de raios $\mathrm{X}$ e pelo método gravimétrico, utilizando três corpos de prova $(50 \times 50 \mathrm{~mm})$ por tratamento. Após as determinações de densidade aparente, os 24 corpos de prova foram submetidos ao ensaio de ligação interna. O método gravimétrico aplicado na determinação da densidade aparente apresentou resultados significativamente maiores do que o método densitométrico. Verificou-se que a densidade mínima do painel, obtida pela densitometria de raios $\mathrm{X}$, teve forte influência na densidade média do painel. Houve correlação entre os valores de densidade aparente dos painéis obtidos por atenuação de raios X e os resultados de ligação interna.
\end{abstract}

Palavras-chave: densitometria de raios $\mathrm{X}$, método gravimétrico, painéis de madeira.

\section{Relationship between Density Profile and Internal Bond of Pinus spp. OSB panels}

\begin{abstract}
This study aimed to determine the density, by gravimetric method and X-ray densitometry, of Oriented Strand Boards (OSB) produced with Pinus taeda and Pinus elliottii var. elliottii x Pinus caribaea var. hondurensis, to obtain densitometric profiles and observe their correlation with the internal bond. The average density of the panels produced was determined by the methodology of X-ray densitometry and the gravimetric method, using 3 specimens $(50 \times 50$ $\mathrm{mm})$ per treatment. After apparent density measurement, the 24 specimens were subjected to the internal bond test. The gravimetric method applied for the determination of apparent density of the panels presented significantly higher results compared with the densitometric method. It was possible to observe that the minimum density of the board, obtained by the method of X-ray densitometry, has a strong influence on its average density. Correlation was observed between the apparent densities of the panels and the results of internal bond.
\end{abstract}

Keywords: X-ray densitometry, gravimetric method, wood panels. 


\section{INTRODUÇÃO}

A produção de painéis particulados à base de madeira encontra-se em contínua evolução tecnológica; portanto, é de grande importância o estudo dos fatores que possam afetar a produção do painel e a sua qualidade final. Diversos fatores influenciam na adequação das propriedades dos painéis, merecendo destaque a densidade do painel, a qual está relacionada à quantidade de partículas para uma determinada dimensão do painel, a qual influencia significativamente as suas propriedades físico-mecânicas (Maloney, 1993).

Brochmann et al. (2004) relataram que os valores de densidade em painéis OSB são altamente significativos no inchamento em espessura e responsáveis por grande parte da variabilidade nos valores obtidos nos ensaios de ligação interna.

A densidade dos painéis de partículas produzidos por prensagem a quente não se mostra uniforme na direção da sua espessura. Tal variação consiste no chamado perfil de densidade vertical ou gradiente de densidade vertical e pode afetar quase todas as propriedades físicas e mecânicas do produto (Jin et al., 2009). O perfil de densidade vertical de painéis OSB é determinado por diversos fatores, tais como a geometria das partículas, a distribuição da umidade no colchão de partículas, o tempo de fechamento da prensa, a temperatura da prensa, a reatividade da resina e a resistência das partículas de madeira à compressão (Kelly, 1977; Wang et al., 2006; Belini, 2007; Belini et al., 2009, 2010; Chen et al., 2010).

Os valores de densidade aparente dos painéis são usualmente determinados pelo método gravimétrico, o qual consiste na medição direta de todos os corpos de prova dos painéis e relaciona massa pelo volume correspondente. Embora seja tradicionalmente utilizado, tal método não determina pontualmente a variação da densidade ao longo da espessura. Nesse sentido, a técnica de densitometria de raios $\mathrm{X}$ para determinação da densidade aparente tem se destacado devido à precisão, ao detalhamento e à praticidade, com alta resolução espacial (Chen et al., 2010).
A metodologiadedensitometriaderaiosX $\mathrm{X}$ ermite conhecer o efeito das variáveis da fase de prensagem das camadas de partículas e deve ser considerada como análise de rotina no controle de qualidade dos painéis, em escala de laboratório e industrial, permitindo calibrar os ciclos de prensagem aplicados em linha de produção pela análise dos perfis em laboratório. Os raios $\mathrm{X}$ consistem de uma radiação eletromagnética penetrante e invisível a olho nu, e quando atravessam a madeira, há uma interação, na qual parte dos fótons de raios $\mathrm{X}$ é removida do feixe incidente numa quantidade proporcional à massa específica da madeira, da espessura da amostra e do número de fótons incidentes (Siqueira, 2004).

Chen \& Wellwood (2002) e Wang et al. (2007) utilizaram um sistema de raios X para analisar a distribuição de densidade de painéis OSB comerciais. Os autores concluíram que a metodologia permite uma amostragem mais ampla na caracterização dos efeitos da densidade sobre outras propriedades dos painéis.

Nesse sentido, o presente trabalho teve por objetivos determinar a densidade aparente dos painéis OSB de Pinus taeda e Pinus elliottii var. elliottii $\times$ Pinus caribaea var. hondurensis pelo método gravimétrico e pela densitometria de raios $\mathrm{X}$, obter os perfis de densidade dos painéis OSB pela densitometria de raios $\mathrm{X}$ e testar a relação desse método com a propriedade mecânica de ligação interna.

\section{MATERIAL E MÉTODOS}

\subsection{Delineamento experimental}

O delineamento experimental (Tabela 1) constituiu-se de oito tratamentos. Para cada um dos tratamentos, foram produzidos três painéis OSB, com densidade nominal de $0,65 \mathrm{~g} / \mathrm{cm}^{3}$ e dimensões de $560 \times 560 \times 15,7 \mathrm{~mm}$. As partículas do tipo strand foram produzidas nas dimensões de 0,65 $\times 25 \times 90 \mathrm{~mm}$ (espessura, largura e comprimento, respectivamente), a partir de cinco árvores do híbrido Pinus elliottii var. elliottii $\times$ Pinus caribaea var. hondurensis $(\mathrm{PECH})$ e quatro árvores da espécie Pinus taeda L. (PT) 


\subsection{Manufatura dos painéis OSB}

$\mathrm{O}$ adesivo utilizado foi o fenol-formaldeído (FF), com teor de sólidos de 53,2\%, pH igual a 11,74, gel time a $121^{\circ} \mathrm{C}$ de 8,33 minutos e viscosidade a $25^{\circ} \mathrm{C}$ de $620 \mathrm{cP}$. A quantidade de adesivo empregada foi de $6 \%$ de sólidos (base massa seca das partículas). Foi adicionada parafina na quantidade de $1 \%$ de sólidos (base massa seca das partículas).

Todos os painéis OSB foram produzidos com três camadas, constituídas por face, miolo e face, nas respectivas proporções de 30:40:30.

Após o colchão de partículas sofrer uma préprensagem a frio, este foi colocado em uma prensa hidráulica para prensagem a quente. A Figura 1 ilustra os dois ciclos de prensagem (convencional e modificado) adotados. Em ambos os ciclos de prensagem, a pressão máxima foi de $35 \mathrm{kgf} / \mathrm{cm}^{2}$, a temperatura de $180^{\circ} \mathrm{C}$ e o tempo total igual a 10 minutos, sendo utilizados separadores com espessura de $15,7 \mathrm{~mm}$.

\subsection{Determinação da densidade aparente dos painéis OSB por densitometria de raios $X$}

Dos 24 painéis OSB produzidos, foram retirados corpos de prova $(50 \times 50 \mathrm{~mm})$, os quais foram mantidos em câmara de climatização (temperatura de $22 \pm 2^{\circ} \mathrm{C}$ e umidade relativa de $65 \pm 5 \%$ ) e, em seguida, analisados em densitômetro de raios X marca QMS modelo QDP-01X (Figura 2a). As amostras (50 $\times 50 \mathrm{~mm}$ ) foram encaixadas no suporte metálico que continha um porta-amostras e transferidas para um compartimento interno blindado do equipamento, seguindo-se a sua calibração e o escaneamento (varredura) contínuo por um feixe de raios $\mathrm{X}$ colimado (Figura 2b). Os valores de raios $\mathrm{X}$ que atravessaram os painéis OSB foram transformados

Tabela 1. Delineamento experimental para a produção dos painéis OSB.

Table 1. Experimental design for the production of OSB panels.

\begin{tabular}{|c|c|c|c|}
\hline Tratamentos & $\begin{array}{c}\text { Proporção de partículas } \\
\text { de PECH }(\%)\end{array}$ & $\begin{array}{c}\text { Proporção de partículas } \\
\text { de PT (\%) }\end{array}$ & Ciclo de Prensagem \\
\hline T1 & 0 & 100 & \multirow{4}{*}{ Convencional } \\
\hline T2 & 100 & 0 & \\
\hline T3 & 75 & 25 & \\
\hline T4 & 50 & 50 & \\
\hline T5 & 0 & 100 & \multirow{4}{*}{ Modificado } \\
\hline T6 & 100 & 0 & \\
\hline T7 & 75 & 25 & \\
\hline T8 & 50 & 50 & \\
\hline
\end{tabular}
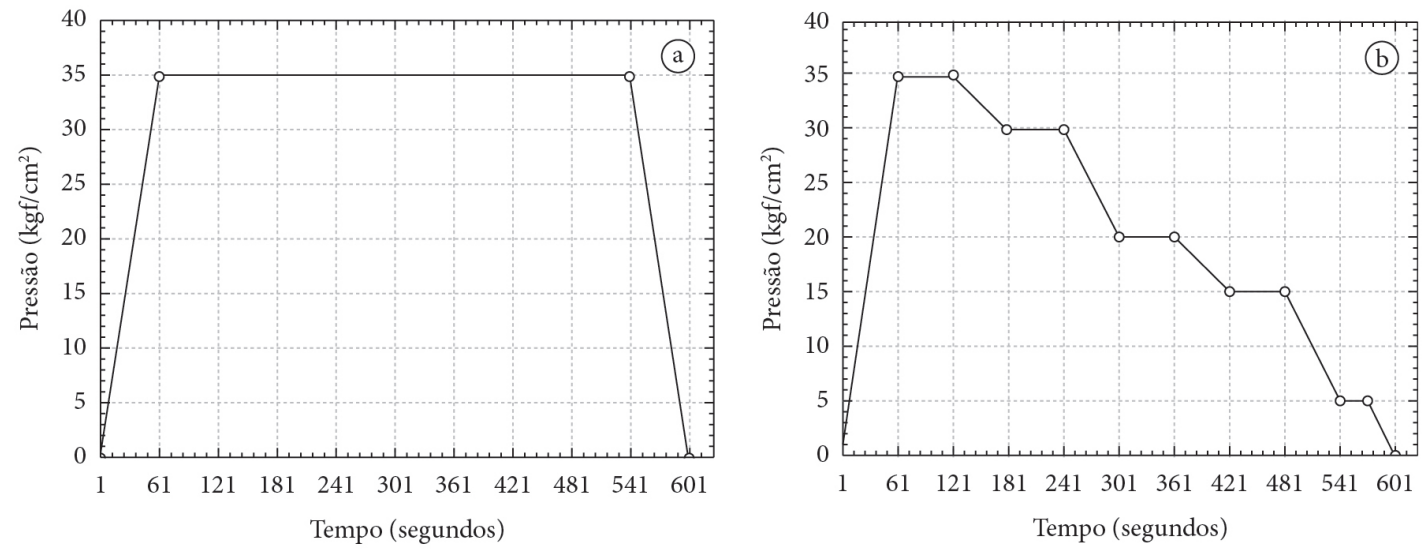

Figura 1. Ciclos de prensagem: a) Ciclo de prensagem convencional; b) Ciclo de prensagem modificado. Figure 1. Pressing cycles: a) Conventional pressing cycle; b) Modified pressing cycle. 

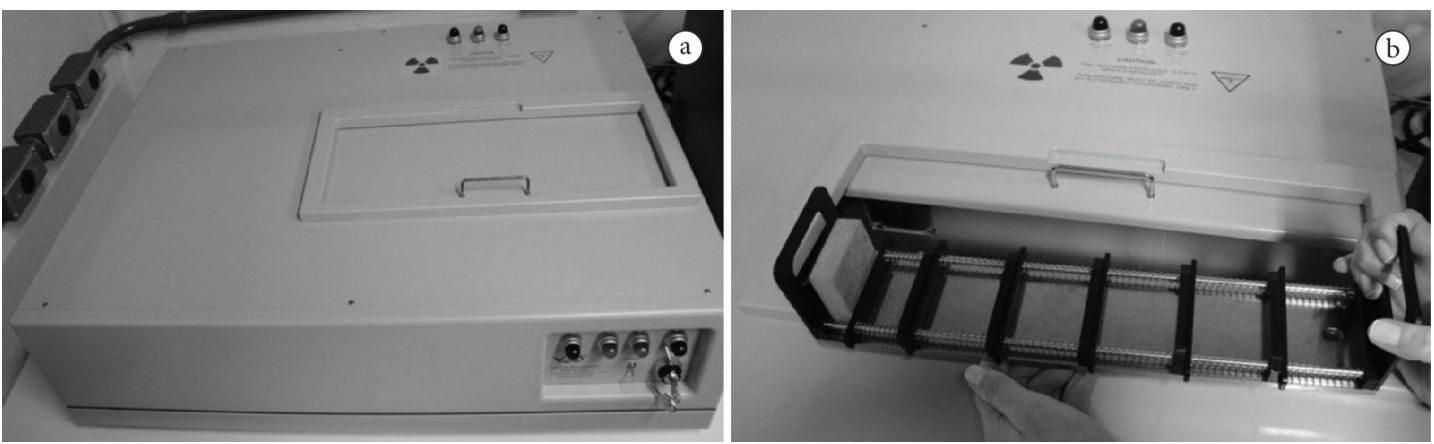

Figura 2. Obtenção dos perfis densitométricos dos painéis OSB por atenuação de raios X. a) Equipamento de raios X QDP-01X; b) Encaixe do suporte metálico com porta-amostras no compartimento de leitura.

Figure 2. Obtaining density profiles of OSB. a) X-ray equipment QDP-01X; b) Compartment reading with the samples.

em densidade aparente por meio do software QMS (Quintek Measurement Systems, 1999).

Os valores de densidade aparente foram determinados a cada $40 \mu \mathrm{m}$ ao longo da espessura dos 24 painéis OSB (uma amostra por painel). Os perfis foram caracterizados pela determinação de três diferentes parâmetros de densidade: (i) densidade média, (ii) densidade máxima e (iii) densidade mínima do painel.

\subsection{Determinação da densidade aparente pelo método gravimétrico por medição direta}

A massa específica de cada painel produzido foi determinada através da medição direta de 24 corpos de prova (uma amostra por painel), obtendo-se as suas massas ( $\mathrm{g}$ ) através de balança de precisão e suas dimensões (largura, comprimento e espessura) através de paquímetro. Por meio da multiplicação das dimensões dos corpos de prova, foram obtidos os seus volumes $\left(\mathrm{cm}^{3}\right)$. Finalmente, a massa específica $\left(\mathrm{g} / \mathrm{cm}^{3}\right)$ de cada corpo de prova foi obtida pela divisão da sua massa pelo volume correspondente (ASTM, 2006).

\subsection{Determinação da ligação interna}

Após as determinações de densidade aparente, os 24 corpos de prova $(50 \times 50 \mathrm{~mm})$ foram submetidos ao ensaio de ligação interna. Para a realização do ensaio mecânico, foi utilizada uma máquina universal de ensaios com capacidade para aplicação de força de até 30 toneladas. Os procedimentos de ensaio empregados na determinação da propriedade ligação interna foram baseados na norma ASTM D 1037 (2006).

\subsection{Análises estatísticas}

Os dados obtidos foram submetidos ao teste de normalidade de Shapiro-Wilk e, em seguida, para verificar o efeito dos métodos de densitometria de raios $\mathrm{X}$ e gravimétrico na determinação da densidade aparente dos painéis OSB, foi aplicado o teste de Tukey, conduzido no nível de $5 \%$ de probabilidade de erro. Foi realizada uma análise de regressão múltipla (stepwise), visando a selecionar a variável que mostrou maior influência na densidade aparente média dos painéis. Todas as correlações foram determinadas a partir do Coeficiente de Correlação de Pearson (r), com a utilização do programa SPSS.

\section{RESULTADOS E DISCUSSÃO}

\subsection{Valores médios de densidade aparente dos painéis OSB}

Os valores médios de densidade aparente dos painéis OSB, obtidos pelos métodos de densitometria de raios $\mathrm{X}$ e gravimétrico, para cada tratamento, encontram-se na Tabela 2.

$\mathrm{Na}$ análise da densidade aparente média, máxima e mínima dos painéis (Tabela 2), obtidas pela atenuação de raios $\mathrm{X}$ e pelo método gravimétrico, não foi observada diferença significativa entre os tratamentos, mostrando que a formação do colchão foi homogênea, não havendo irregularidade no 
Tabela 2. Valores médios de densidade aparente dos painéis OSB.

Table 2. Average values of apparent density of the OSB panels.

\begin{tabular}{|c|c|c|c|c|}
\hline \multirow{3}{*}{ Tratamento } & \multicolumn{3}{|c|}{ Densidade aparente (densitometria de raios X) } & \multirow{2}{*}{$\begin{array}{l}\text { Densidade aparente } \\
\text { (método gravimétrico) }\end{array}$} \\
\hline & Média & Máxima & Mínima & \\
\hline & \multicolumn{4}{|c|}{$\left(\mathrm{g} / \mathrm{cm}^{3}\right)$} \\
\hline $100 \% \mathrm{PT} / \mathrm{CC}$ & $0,64 a^{1}(0,05)^{2}$ & $0,72 \mathrm{a}(0,06)$ & $0,55 \mathrm{a}(0,05)$ & 0,68 a $(0,01)$ \\
\hline $100 \% \mathrm{PECH} / \mathrm{CC}$ & 0,59 a $(0,03)$ & 0,70 a $(0,06)$ & 0,49 a $(0,02)$ & 0,61 a $(0,01)$ \\
\hline $75 \% \mathrm{PECH}+25 \% \mathrm{PT} / \mathrm{CC}$ & $0,64 \mathrm{a}(0,05)$ & 0,81 a $(0,05)$ & $0,51 \mathrm{a}(0,06)$ & 0,67 a $(0,05)$ \\
\hline $50 \% \mathrm{PECH}+50 \% \mathrm{PT} / \mathrm{CC}$ & 0,58 a $(0,06)$ & 0,73 a $(0,07)$ & 0,46 a $(0,07)$ & 0,63 a $(0,04)$ \\
\hline $100 \% \mathrm{PT} / \mathrm{CM}$ & 0,68 a $(0,03)$ & $0,78 \mathrm{a}(0,02)$ & 0,59 a $(0,03)$ & $0,73 \mathrm{a}(0,01)$ \\
\hline $100 \% \mathrm{PECH} / \mathrm{CM}$ & 0,65 a $(0,11)$ & $0,80 \mathrm{a}(0,12)$ & $0,55 \mathrm{a}(0,14)$ & 0,68 a $(0,08)$ \\
\hline $75 \% \mathrm{PECH}+25 \% \mathrm{PT} / \mathrm{CM}$ & $0,57 \mathrm{a}(0,05)$ & $0,70 \mathrm{a}(0,05)$ & $0,46 \mathrm{a}(0,04)$ & 0,63 a $(0,05)$ \\
\hline $50 \% \mathrm{PECH}+50 \% \mathrm{PT} / \mathrm{CM}$ & 0,63 a $(0,04)$ & $0,75 \mathrm{a}(0,06)$ & 0,52 a $(0,05)$ & 0,68 a $(0,07)$ \\
\hline Média Geral & $0,62(0,06)$ & $0,75(0,07)$ & $0,52(0,07)$ & $0,66(0,05)$ \\
\hline CV (\%) & 9,39 & 9,11 & 12,68 & 7,31 \\
\hline
\end{tabular}

${ }^{1}$ Médias seguidas de mesma letra, na coluna, não diferem estatisticamente pelo teste de Tukey $(\alpha=0,05) .{ }^{2}$ Valores entre parênteses correspondem ao desvio padrão $\left(\mathrm{g} / \mathrm{cm}^{3}\right)$. $\mathrm{PT}=$ Pinus taeda $; \mathrm{PECH}=$ Pinus elliottii var. elliottii $\times$ Pinus caribaea var. hondurensis; $\mathrm{CC}=$ Ciclo Convencional; $\mathrm{CM}=$ Ciclo Modificado .

processo de deposição das partículas ao longo dos painéis.

De acordo com a Tabela 3, o método gravimétrico, aplicado na determinação da densidade aparente dos painéis, diferiu significativamente do método densitométrico (leituras micrométricas no sentido da espessura). Os menores valores no método de densitometria de raios $\mathrm{X}$ podem estar associados ao fato de que o feixe colimado permite uma atenuação micrométrica no sentido da espessura do painel, determinando, assim, a densidade aparente pontual em função da geometria da partícula, do teor de umidade e da reatividade da resina.

\subsection{Correlação entre os métodos de obtenção de densidade aparente}

As correlações da densidade aparente máxima e mínima com a densidade média obtidas pela densitometria de raios $\mathrm{X}$ foram significativas (no nível de $1 \%$ ) e positivas (Tabela 4 ). O teste mostrou um alto valor de correlação entre a densidade aparente obtida por ambos os métodos (densitometria de raios $\mathrm{X}$ e gravimétrico).

Os resultados da análise de regressão múltipla para analisar a relação da densidade aparente média dos painéis e as demais componentes - densidades aparentes máxima e mínima obtidas pela densitometria de raios $\mathrm{X}$, bem como a sua ordem de importância, considerando os valores
Tabela 3. Densidade aparente média dos painéis OSB obtida pelos métodos gravimétrico e densitometria de raios $\mathrm{X}$.

Table 3. Average apparent density of the OSB panels obtained by gravimetric method and X-ray densitometry.

\begin{tabular}{|cc|}
\hline Método & $\begin{array}{r}\text { Densidade aparente } \\
\left(\mathbf{g} / \mathbf{c m}^{\mathbf{3}}\right)\end{array}$ \\
\hline Densitometria de raios X & $0,62 \mathrm{a}$ \\
\hline Gravimétrico (medição direta) & $0,66 \mathrm{~b}$ \\
\hline Média geral & 0,64 \\
\hline CV (\%) & 9,02 \\
\hline
\end{tabular}

Médias seguidas de mesma letra não diferem estatisticamente pelo teste de Tukey $(\alpha=0,05)$.

Tabela 4. Correlação de Pearson para os valores médios de densidade aparente obtida pelos métodos gravimétrico e densitometria de raios $\mathrm{X}$.

Table 4. Pearson correlation for the average values of apparent density obtained by gravimetric method and $\mathrm{X}$-ray densitometry.

\begin{tabular}{ccccc} 
& $\mathbf{D}_{\text {média }}$ & $\mathbf{D}_{\text {mínima }}$ & $\mathbf{D}_{\text {máxima }}$ & $\mathbf{D}_{\text {gravimétrico }}$ \\
$\mathbf{D}_{\text {média }}$ & 1 & & & \\
$\mathbf{D}_{\text {mínima }}$ & $0,956^{* *}$ & 1 & & \\
$\mathbf{D}_{\text {máxima }}$ & $0,903^{* *}$ & $0,774^{* *}$ & 1 & \\
$\mathbf{D}_{\text {gravimétrico }}$ & $0,870^{* *}$ & $0,845^{* *}$ & $0,740^{* *}$ & 1 \\
\hline
\end{tabular}

${ }^{*}$ Correlação significativa no nível de $1 \%$; $\mathrm{D}=$ densidade aparente.

médios das três repetições de cada um dos oito tratamentos - encontram-se na Tabela 5. Observouse que a variável selecionada para explicar a densidade aparente média dos painéis, analisada 
Tabela 5. Análise de regressão múltipla entre a densidade aparente média e suas componentes, densidade máxima e mínima.

Table 5. Multiple regression analysis between average apparent density and its components, maximum and minimum density.

\begin{tabular}{cccc} 
Passos & Dmín & Dmáx & $\mathbf{R}^{\mathbf{2}}$ \\
\hline 1 & $64,0^{*}$ & 41,0 & 0,98 \\
2 & 95,6 & NS & 0,91 \\
\hline
\end{tabular}

* Os valores colocados abaixo das variáveis independentes são os coeficientes estandarizados (betas) expressos em porcentagem e representam a proporção com que cada variável contribui para a determinação da variável dependente, explicada pela regressão; NS - Indica que a variável independente se manifestou não significativa para o nível de probabilidade de $\mathrm{p}<0,05 ;$ Dmín $=$ Densidade mínima; Dmáx $=$ Densidade máxima.

em regressão stepwise, a $5 \%$ de probabilidade, foi a densidade mínima, compondo o modelo de regressão (Equação 1), que explica 91\% da variação total da densidade aparente média, com a densidade mínima responsável por 95,6\% da variação.

$D_{\text {média }}=0,195+\left(0,828 \times D_{\text {mínima }}\right)$

\subsection{Perfis de densidade aparente dos painéis OSB por densitometria de raios $X$}

Os perfis de densidade aparente dos painéis OSB obtidos pelo método de densitometria de raios $\mathrm{X}$, para cada tratamento, encontram-se na Figura 3.

Os painéis OSB, independentemente do ciclo de prensagem, apresentaram maiores valores de densidade nas faces e menores valores no miolo, formando um perfil característico, em forma de letra ' $M$ '. Este formato dos perfis de densidade ao longo da espessura dos painéis OSB é característico para painéis de madeira e afeta quase todas as propriedades físicas e mecânicas (Sicoplan, 1996; Suzuki \& Miyamoto, 1998; Eleotério, 2000; Ayrilmis, 2007). Conforme Wong et al. (1999), durante o processo de prensagem a quente, a interação entre calor, umidade e pressão dá origem à deformação não uniforme dos elementos, o que resulta numa distribuição desigual de densidade ao longo da espessura do painel. Strickler (1959) e Suchsland \& Woodson (1986) atribuem as diferenças de densidade entre faces e miolo aos seguintes aspectos: configuração das partículas; compressão, conteúdo e distribuição de umidade; temperatura e velocidade de fechamento da prensa, bem como à pressão e à duração do ciclo de prensagem a quente.

Segundo Belini et al. (2009), o perfil de densidade determinado por meio dos raios X é importante para a recomendação da aplicação do painel OSB e para aferir as condições de prensagem direcionadas para a confecção de painéis de melhor desempenho, em aplicações específicas.

\subsection{Ligação interna}

Os valores médios de ligação interna, para cada tratamento, estão apresentados na Tabela 6 .

De acordo com a análise de variância efetuada para a variável ligação interna dos painéis OSB do presente estudo, verifica-se que não houve diferença significativa entre os tratamentos. A norma CSA 0437 (1993) estipula o valor mínimo para a propriedade de ligação interna igual a 0,34 $\mathrm{MPa}$, para ambas as classificações O1 e O2. Com base nos dados da Tabela 6, é possível verificar que os painéis OSB de todos os tratamentos exibiram valores médios de ligação interna acima do mínimo requerido pela norma em referência.

Mendes et al. (2013), avaliando as propriedades de painéis OSB produzidos com a madeira de Pinus taeda L. - massa específica nominal de 0,65 g/ $\mathrm{cm}^{3}$, colados com $6 \%$ de adesivo à base de fenolformaldeído -, obtiveram valor médio de 0,60 $\mathrm{MPa}$ para a propriedade ligação interna, ou seja, um valor relativamente superior ao obtido para os painéis produzidos com partículas 100\% PT (Tabela 6).

Saldanha \& Iwakiri (2009), avaliando painéis OSB produzidos com a madeira de Pinus taeda L. - densidade nominal de $0,65 \mathrm{~g} / \mathrm{cm}^{3}$ e $6 \%$ de adesivo fenol-formaldeído -, obtiveram o valor médio de 0,39 MPa para a propriedade ligação interna, valor inferior aos obtidos no presente trabalho.

\subsection{Correlação entre ligação interna e} densidade aparente obtida pela densitometria de raios $X$

Os valores de densidade aparente ao longo do perfil foram correlacionados, por meio do coeficiente de correlação de Pearson, com a propriedade mecânica ligação interna, conforme Tabela 7 . 

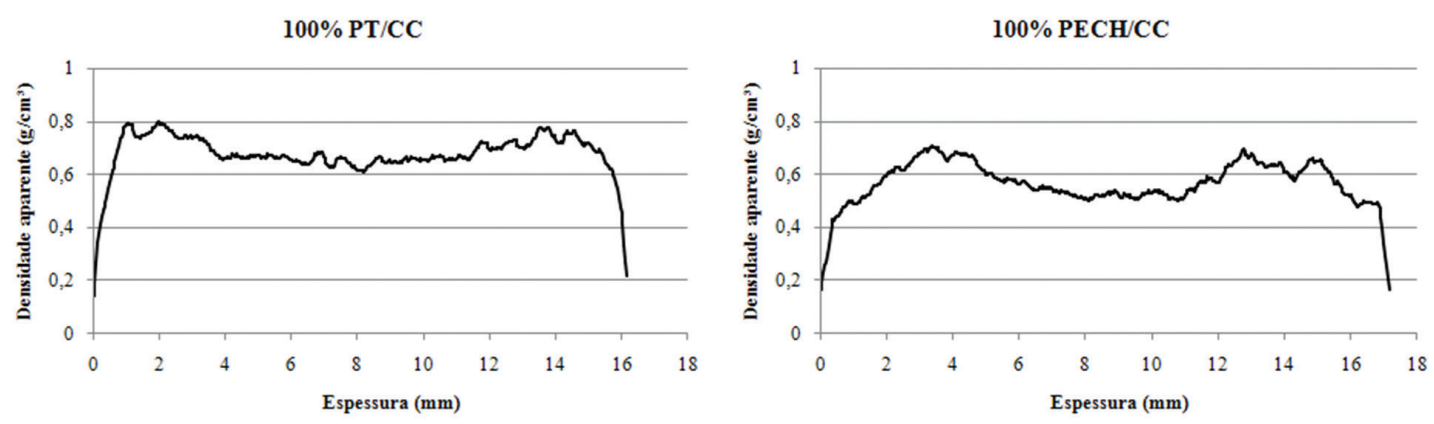

$75 \% \mathrm{PECH}+25 \% \mathrm{PT} / \mathrm{CC}$
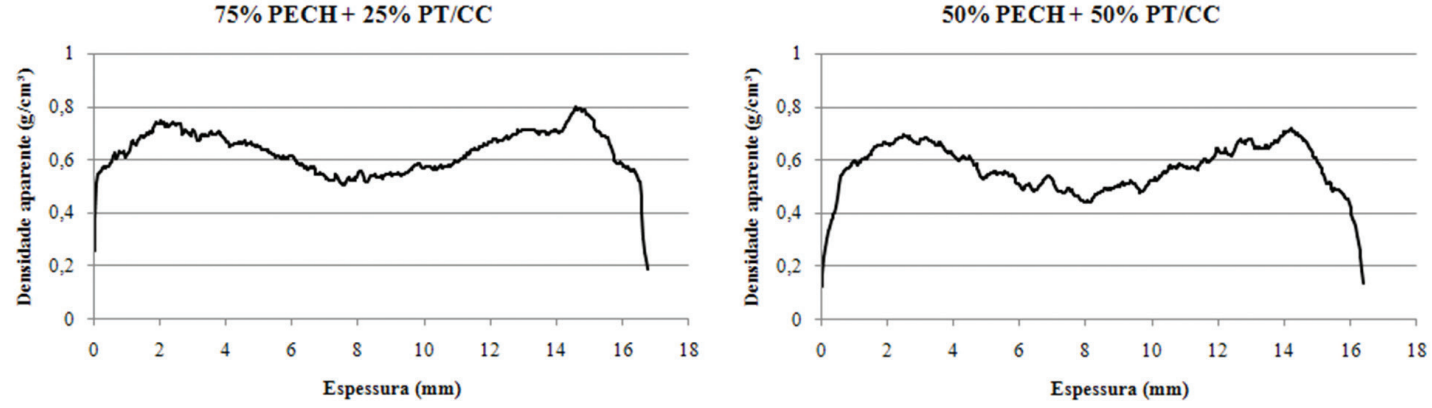

$100 \% \mathrm{PT} / \mathrm{CM}$

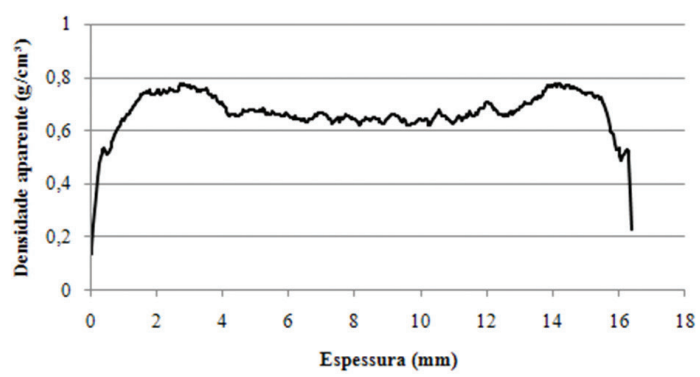

$100 \% \mathrm{PECH} / \mathrm{CM}$

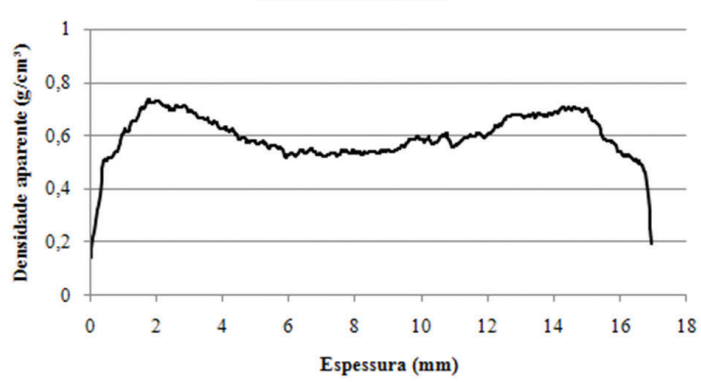

$75 \% \mathrm{PECH}+25 \% \mathrm{PT} / \mathrm{CM}$
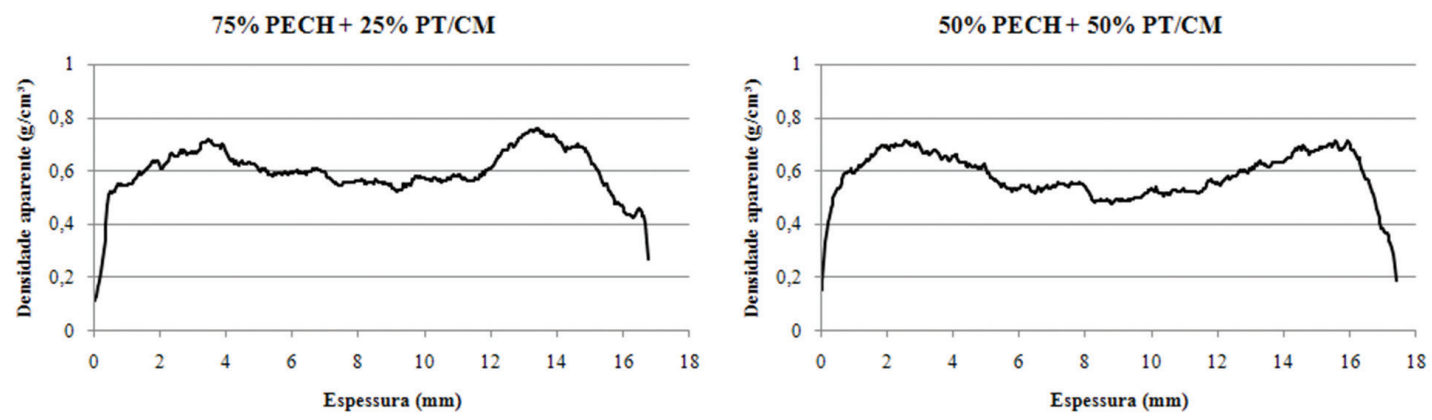

Figura 3. Perfis de densidade aparente ao longo da espessura dos painéis OSB.

Figure 3. Apparent density profiles of the OSB panels in the thickness direction.

A propriedade ligação interna apresentou correlação com as densidades aparentes média, mínima, máxima e das camadas interna e externas na faixa de 0,71 a 0,82 , indicando que houve influência da densidade aparente dos painéis sobre os resultados dessa propriedade. De acordo com Cloutier (1998), a resistência à ligação interna é em grande parte determinada pela menor densidade que ocorre na camada interna. Wong et al. (1999) e Jin et al. (2009), estudando propriedades de painéis de partículas 
Tabela 6. Valores médios de ligação interna dos painéis OSB.

Tabela 6. Average values of internal bond of the OSB panels.

\begin{tabular}{|cc|}
\hline Tratamento & Ligação Interna $(\mathbf{M P a})$ \\
\hline $100 \% \mathrm{PT} / \mathrm{CC}$ & $0,51 \mathrm{a}^{1}(0,11)^{2}$ \\
\hline $100 \% \mathrm{PECH} / \mathrm{CC}$ & $0,34 \mathrm{a}(0,06)$ \\
\hline $75 \% \mathrm{PECH}+25 \% \mathrm{PT} / \mathrm{CC}$ & $0,50 \mathrm{a}(0,08)$ \\
\hline $50 \% \mathrm{PECH}+50 \% \mathrm{PT} / \mathrm{CC}$ & $0,41 \mathrm{a}(0,11)$ \\
\hline $100 \% \mathrm{PT} / \mathrm{CM}$ & $0,54 \mathrm{a}(0,19)$ \\
\hline 100\% PECH/CM & $0,52 \mathrm{a}(0,08)$ \\
\hline $75 \% \mathrm{PECH}+25 \% \mathrm{PT} / \mathrm{CM}$ & $0,44 \mathrm{a}(0,09)$ \\
\hline $50 \% \mathrm{PECH}+50 \% \mathrm{PT} / \mathrm{CM}$ & $0,44 \mathrm{a}(0,08)$ \\
\hline Média Geral & $0,46(0,11)$ \\
\hline CV $(\%)$ & 24,21 \\
\hline
\end{tabular}

${ }^{1}$ Médias seguidas de mesma letra, na coluna, não diferem estatisticamente pelo teste de Tukey $(\alpha=0,05) .{ }^{2}$ Valores entre parênteses correspondem ao desvio padrão $(\mathrm{MPa}) . \mathrm{PT}=$ Pinus taeda PECH $=$ Pinus elliottii var. elliottii $\times$ Pinus caribaea var. hondurensis; $\mathrm{CC}=$ Ciclo Convencional; $\mathrm{CM}=$ Ciclo Modificado.

Tabela 7. Correlação de Pearson entre a ligação interna e as densidades obtidas pela densitometria de raios $\mathrm{X}$ dos painéis OSB.

Table 7. Pearson Correlation between internal bond and density obtained by X-ray densitometry of the OSB panels.

\begin{tabular}{lccccc} 
& $\mathbf{D}_{\text {Média }}$ & $\mathbf{D}_{\text {Minima }}$ & $\mathbf{D}_{\text {Máxima }}$ & $\mathbf{D}_{\text {Camada Interna }}$ & $\mathbf{D}_{\text {Camadas Externas }}$ \\
$\begin{array}{r}\text { Ligação } \\
\text { Interna }\end{array}$ & $0,821^{*}$ & $0,735^{*}$ & $0,711^{*}$ & $0,788^{*}$ & $0,817^{*}$ \\
\hline
\end{tabular}

* Significativo no nível de $5 \%$ de probabilidade; $\mathrm{D}=$ densidade aparente.

com diferentes perfis de densidade, concluíram que a ligação interna possui alta correlação com o miolo dos painéis.

Pelo fato de os corpos de prova romperem-se na camada interna, esperava-se que a correlação com a densidade mínima e/ou densidade da camada interna se destacasse.

\section{CONCLUSÕES}

A densitometria de raios $\mathrm{X}$ aplicada na determinação da densidade aparente dos painéis OSB apresentou resultados médios menores em relação ao método gravimétrico, para todos os tratamentos.

Os perfis densitométricos dos painéis OSB, independentemente do ciclo de prensagem e da proporção de partículas de Pinus taeda e Pinus elliottii var. elliottii $\times$ Pinus caribaea var. hondurensis, apresentaram maiores valores de densidade nas faces e menores valores no miolo, formando um perfil característico, em forma de letra ' $M$ '.

A densidade aparente e o formato dos perfis densitométricos dos painéis exerceram influência sobre os resultados de ligação interna.

\section{STATUS DA SUBMISSÃO}

Recebido: 18 out., 2013

Aceito: 12 mar., 2014

Publicado: 28 ago., 2014

\section{AUTOR PARA CORRESPONDÊNCIA}

\section{Paula Gabriella Surdi}

Departamento de Ciências Florestais, Escola Superior de Agricultura Luiz de Queiroz - ESALQ, Universidade de São Paulo - USP, CEP 13418-900, Piracicaba, SP, Brasil

e-mail: paulasurdi@usp.br

\section{REFERENNCIAS}

American Society for Testing Materials - ASTM. ASTM $D$-1037: Standard methods of evaluating the properties of wood-base fiber and particle materials. Philladelphia: ASTM; 2006. p. 1-30. Annual Book of ASTM Standards.

Ayrilmis N. Effect of panel density on dimensional stability of medium and high density fiberboards. Journal of Materials Science 2007; 42: 8551-8557. http:// dx.doi.org/10.1007/s10853-007-1782-8

Belini UL. Caracterização e alterações na estrutura anatômica da madeira do Eucalyptus grandis em três condições de desfibramento e efeito nas propriedades tecnológicas de painéis MDF [tese]. Piracicaba: Universidade de São Paulo; 2007.

Belini UL, Tomazello Filho M, Chagas MP.Densitometria de raios $\mathrm{X}$ aplicada na avaliação tecnológica de painéis MDF de eucalipto. Scientia Forestalis 2009; 37(84): $343-$ 350 .

Belini UL, Zegarra BEV, Tomazello Filho M, Bortoletto Jr G. Densitometria de raios X aplicada em painéis MDF e OSB de Eucalipto e de Pinus. In: Anais do Encontro Brasileiro em Madeiras e em Estruturas de Madeira; 2010; Lavras. Lavras: Universidade Federal de Lavras. CD-ROM. 
Brochmann J, Edwardson C, Shmulsky R. Influence of resin type and flake thickness on properties of OSB. Forest Products Journal 2004; 54(3): 51-55.

Canadian Standards Association - CSA. CSA 0437-0: OSB and waferboard. Ontario; 1993. 18 p.

Chen S, Xiaohong L, Fang L, Wellwood R. Digital X-ray analysis of density distribution characteristics of woodbased panels. Wood Science and Technology 2010; 44(1): 85-93. http://dx.doi.org/10.1007/s00226-009-0256-3

Chen S, Wellwood R. Nondestructive evaluation of oriented strand board. In: Proceedings of the 13th International Symposium on Nondestructive Testing of Wood; 2002; Berkeley. Berkeley; 2002.

Cloutier A. Oriented strandboard (OSB): raw material, manufacturing process, properties of wood-base fiber and particle materials. In: International Seminar on Solid Wood Products of High Technology; 1998; Belo Horizonte. SIF; 1998. p. 173-185.

Eleotério JR. Propriedades físicas e mecânicas de painéis MDF de diferentes densidades e teores de resina [dissertação]. Piracicaba: Universidade de São Paulo; 2000 .

Jin J, Dai C, Hsu WE, Yu C. Properties of strand boards with uniform and conventional vertical density profiles. Wood Science and Technology 2009; 43:559-574. http:// dx.doi.org/10.1007/s00226-009-0248-3

Kelly MW. Critical literature review of relationships between processing parameters and physical properties of particleboard. Madison: Forest Products Laboratory; 1977. USDA For. Serv. Gen. Tec. Rep.

Maloney, T. M. Modern particleboard e dry-process fiberboard manufacturing. 2nd ed. São Francisco: Miller Freeman Publication; 1993. 689 p.

Mendes RF, Bortoletto Jr G, Almeida NF, Surdi PG, Barbeiro IN. Effect of thermal treatment on properties of OSB panels. Wood Science and Technology 2013; 47(2): 243-256. http://dx.doi.org/10.1007/s00226-0120494-7

Quintek Measurement Systems. QMS: Density Profiler Model QDP-01X. Users Guide. Knoxville; 1999.

Saldanha LK, Iwakiri S. Influência da densidade e do tipo de resina nas propriedades tecnológicas de painéis OSB de Pinus taeda L. Floresta 2009; 39(3): 571-576.

Sicoplan. MDF plant: technology manual. v. 1. 1996.

Siqueira KP. Variabilidade da massa específica de Pinus taeda L. em diferentes classes de sítio [dissertação]. Curitiba: Universidade Federal do Paraná; 2004.

Strickler MD. Effect of press cycles and moisture content on properties of douglas-fir flakeboard. Forest Products Journal 1959; 7: 203-215.

Suchsland O, Woodson GE. Fiberboard manufacturing practices in the United States. USDA Forest Service 1986; 112-158.

Suzuki S, Miyamoto K. Effect of manufacturing parameters on the linear expansion and density profile of particleboard. Journal of Wood Science 1998; 44:444450. http://dx.doi.org/10.1007/BF00833408

Wang X, Salenikovich A, Mohammad M. Localized density effects on fastener holding capacities in woodbased panels. Forest Products Journal 2007; 57(12): 103-109.

Wang X, Mohammad M, Hu LJ, Salenikovich A. Evaluation of density distribution in wood-based panels using X-ray scanning. E-Journal of Nondestructive Testing 2006; 11(4).

Wong ED, Zhang M, Wang Q, Kawai S. Formation of the density profile and its effects on the properties of particleboard. Wood Science and Technology 1999; 33: 327-340. http://dx.doi.org/10.1007/s002260050119 